\title{
Magnetohydrodynamics and electron-electron interaction of massless Dirac fermions
}

\author{
D. A. Khudaiberdiev $\odot,{ }^{1,2}$ G. M. Gusev $\odot,{ }^{3}$ E. B. Olshanetsky, ${ }^{1}$ Z. D. Kvon, ${ }^{1,2}$ and N. N. Mikhailov ${ }^{1,2}$ \\ ${ }^{1}$ Institute of Semiconductor Physics, Novosibirsk 630090, Russia \\ ${ }^{2}$ Novosibirsk State University, Novosibirsk 630090, Russia \\ ${ }^{3}$ Instituto de Física da Universidade de São Paulo, 135960-170, São Paulo, SP, Brazil
}

(Received 7 April 2021; revised 12 July 2021; accepted 15 July 2021; published 30 July 2021)

\begin{abstract}
The magnetotransport properties of massless Dirac fermions in a gapless HgTe quantum well are investigated. In samples with narrow channels, a large negative magnetoresistance with a Lorentzian profile is observed, which is interpreted as a manifestation of electron viscosity due to an electron-electron interaction. A comparison of experiment with theory yields the shear stress relaxation time of the Dirac fermions caused by electron-electron scattering.
\end{abstract}

DOI: 10.1103/PhysRevResearch.3.L032031

In many cases, for the description of phenomena in solidstate structures, the approximation of noninteracting electrons turns out to be inapplicable and the electron-electron (e-e) interaction, which is one of the oldest problems in solid state physics, acquires a key role [1,2]. The reduction of dimensionality from three dimensions (3D) to two dimensions (2D) sharply enhances the importance of e-e interaction effects [3]. Moreover, it has been discovered recently that the relaxation of perturbations of various types in 2D Fermi systems could be both quantitatively and qualitatively different from the quasiparticle lifetime [4-6].

In the so-called hydrodynamic regime of electron transport the shear stress relaxation time $\tau_{2, e e}$ is responsible for shear viscosity which describes the friction between adjacent layers of liquid moving with different velocities. The index 2 in the e-e scattering time subscript $\tau_{2, e e}$ means that the viscosity coefficient is determined by the relaxation of the second harmonic of the distribution function [6]. The hydrodynamic regime requires $l / l_{2, e e} \gg 1$ and $l_{2, e e} / W \ll 1$, where $l=v_{F} \tau$ is the electron transport mean free path related to momentum relaxation time $(\tau)$ brought about by scattering on defects and phonons, $v_{F}$ is the Fermi velocity, $W$ is the channel width, and $l_{2, e e}=v_{F} \tau_{2, e e}$ is the mean free path for shear viscosity relaxation [7-41]. In the presence of the perpendicular magnetic field $B$ the shear viscosity becomes a tensor depending on $B$, which leads to giant negative magnetoresistance with a Lorentzian profile in narrow channel devices [15]. It is possible to extract shear viscosity and shear stress relaxation time from the comparison of the experimental data and the theory. This has been performed in Refs. [27-30] for highmobility GaAs quantum wells with a parabolic spectrum. The study of the temperature dependence of viscosity allows one to distinguish between the electron hydrodynamics and

Published by the American Physical Society under the terms of the Creative Commons Attribution 4.0 International license. Further distribution of this work must maintain attribution to the author(s) and the published article's title, journal citation, and DOI. ballistic transport [41] and explain the difference between e-e scattering in a single well and in a bilayer [42].

The hydrodynamic approach is valid for systems with a linear spectrum, such as graphene. Moreover, the electron hydrodynamics in graphene has aroused large interest [40] because the observation of negative nonlocal resistance due to current whirlpools [13] and superballistic flow in graphene point contacts [14] open up the opportunity to study the so-called Dirac liquids. The shear stress relaxation time calculation in Refs. [4,5] highlights the subtle effect of the linear dispersion that distinguishes graphene from the usual parabolic semiconductor-based 2D systems.

Contrary to the quasiparticle lifetime in two-dimensional systems which one expects to be expressed as $\tau_{e e} \sim$ $\frac{\hbar E_{F}}{\left[(k T)^{2} \ln \left(E_{F} / k T\right)\right]}$, it has been predicted that $\tau_{2, e e} \sim \frac{\hbar E_{F}}{(k T)^{2}}$ for a Fermi gas and $\tau_{2, e e} \sim \frac{\hbar \ln ^{2}\left(E_{F} / k T\right)}{(k T)^{2}}$ for a strongly interacting Fermi liquid [6,26]. Proportionality coefficients depend on the dispersion relations and are different for graphene and conventional 2D systems [4-6].

It has been demonstrated that, similar to single-layer graphene, a gapless phase of single-valley Dirac fermions exists in symmetric HgTe quantum wells with a critical width $d_{c}=6.3 \mathrm{~nm}[43,44]$. The equation describing the electron linear dispersion relation near the Dirac point is $E(k)=\hbar v_{F} k$, where the Fermi velocity $v_{F}=7 \times 10^{7} \mathrm{~cm} / \mathrm{s}=c / 430(c$ is the light velocity) is close to the Fermi velocity in graphene $v_{F}=c / 300$. Various methods have been proposed for the observation of viscosity in the hydrodynamic regime in graphene $[13,20]$. Although experimental studies of transport in a magnetic field are mainly focused on observing the Hall viscosity effect [21,22], a subsequent analysis of the results obtained may provide more information on the mechanism of electronelectron shear stress scattering of Dirac fermions.

This Letter presents the results of an experimental study of the magnetotransport properties of two-dimensional Dirac fermions in gapless HgTe quantum wells in a wide temperature range. In samples with a small channel width at a high electron concentration, a large negative magnetoresistance with a Lorentzian profile was found, which is in agreement 

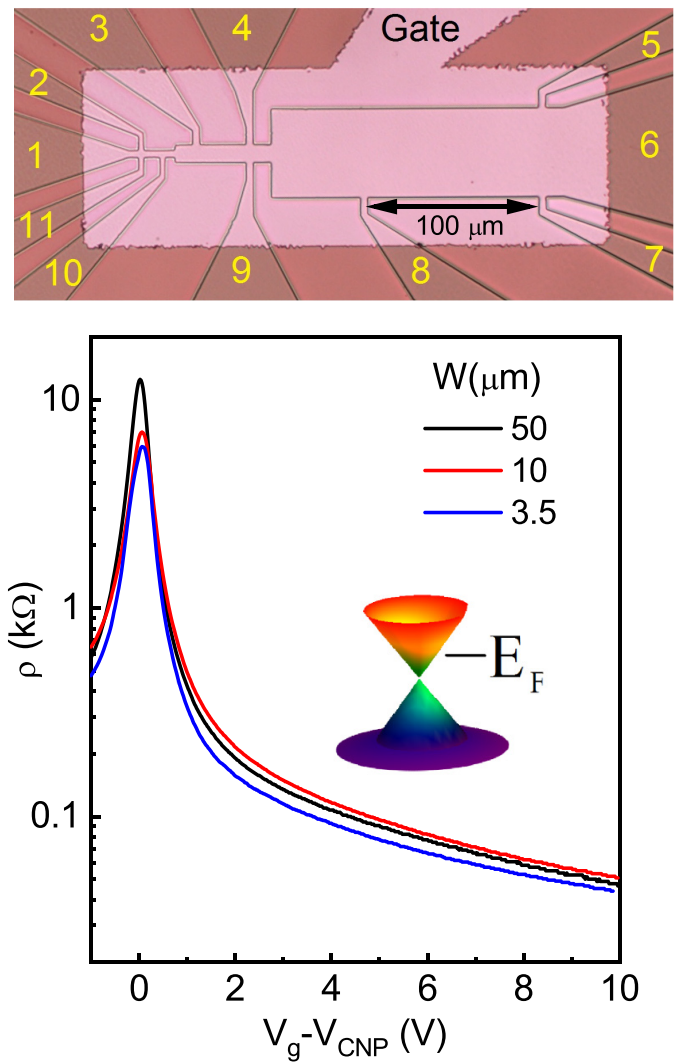

FIG. 1. Schematic of the transistor and the top view of the sample. Resistivity $\rho$ as a function of gate voltage measured for sample sections of different sizes, $T=4.2 \mathrm{~K}$.

with the hydrodynamic model. Comparing experiment with theory, we were able to find the stress relaxation time in a system with a linear spectrum.

$\mathrm{Cd}_{0.65} \mathrm{Hg}_{0.35} \mathrm{Te} / \mathrm{HgTe} / \mathrm{Cd}_{0.65} \mathrm{Hg}_{0.35} \mathrm{Te}$ quantum wells with (013) surface orientations and a well thickness of $6.3 \mathrm{~nm}$ were prepared by molecular beam epitaxy (MBE) [44]. The experimental structures were identical Hall bar devices consisting of three consecutive sections with different widths $W: 3.5,10$, and $50 \mu \mathrm{m}$, respectively. The distance between the voltage probes was $L=12,30,100 \mu \mathrm{m}$, (Fig. 1). In addition, a macroscopic Hall bar device with eight voltage probes was also examined. This Hall bar had a width $W$ of $50 \mu \mathrm{m}$ and three consecutive segments of different lengths $L$ $(100,250,100 \mu \mathrm{m})$ (not shown). A dielectric layer (200 nm of $\mathrm{SiO}_{2}$ ) was deposited on the sample surface and then covered by a TiAu gate. The density variation with gate voltage was $1.1 \times 10^{11} \mathrm{~cm}^{-2} \mathrm{~V}^{-1}$. The resistance in the presence of the perpendicular magnetic field $R(B)$ has been measured in the temperature range $4.2-70 \mathrm{~K}$ using a standard four-point circuit with a 1-13 Hz ac current of 1-10 nA through the sample.

Figure 1 presents the resistivity $\rho=R \frac{W}{L}$ as a function of gate voltage measured at zero magnetic field for segments of different widths. One can see that the resistivity is weakly size dependent and demonstrates a typical peak near the charge neutrality point (CNP) with a value close to $h / 2 e^{2}$. Further, we focus on the sample behavior in the positive gate voltage region, corresponding to the Fermi level residing in the conduction band. In contrast to graphene, the density of states in a zero-gap HgTe quantum well (QW) is not symmetric and it rapidly grows in the valence band when moving away from the Dirac point [44].

Figure 2(a) shows the resistance as a function of magnetic field for two narrow segments of the mesoscopic sample with widths 3.5 and $10 \mu \mathrm{m}$ at $V_{g}=4 \mathrm{~V}$. One can see a large negative magnetoresistance $[R(B)-R(0)<0)]$ with a Lorentzian profile for the $3.5-\mu \mathrm{m}$ segment and a wider triangular-shaped peak for the $10-\mu \mathrm{m}$ segment. The magnetoresistance for the macroscopic sample is shown in the Supplemental Material [45]. It is positive for all gate voltages. The evolution of $R(B)$ with gate voltages in mesoscopic samples is given in Figs. 2(b) and 2(c). One can see that in the vicinity of the $\mathrm{CNP}$ the magnetoresistance becomes positive. Figure 3 shows the representative traces illustrating the evolution of $R(B)$ with temperature for a fixed gate voltage far away from the CNP, $V_{g}=13 \mathrm{~V}$. The resistance curves are almost independent of $T$ at temperatures below $15 \mathrm{~K}$, but $R(B)$ becomes wider at high temperatures. Note that the resistance at $B=0$ increases with $T$. It was found that the $R(T)$ dependences above $4.2 \mathrm{~K}$ can be well described by a cubic law, $R(T) / R(4.2)=1+\alpha_{\text {mes }} T^{3}$, with $\alpha_{\text {mes }}=1.2 \times 10^{-6} \mathrm{~K}^{-3}$. In the macroscopic sample the $R(B=0)$ above $4.2 \mathrm{~K}$ can be approximated with only a quadratic term, $R(T) / R(4.2)=1+\alpha_{\text {macr }} T^{2}$, with $\alpha_{\text {macr }}=$ $8.6 \times 10^{-5} \mathrm{~K}^{-2}$ [45]. The temperature-independent term is described by the interface roughness scattering [46], while the temperature-dependent contribution to the scattering is expected to be due to the phonons, similar to $2 \mathrm{D} \mathrm{GaAs}$ systems [27]. Note, however, that the scattering by acoustic phonons leads to a linear rather than a $T^{2}$ dependence for $T>T_{\mathrm{BG}} \sim$ $4 \mathrm{~K}$, where $T_{\mathrm{BG}}$ is the Bloch-Grüneisen temperature [47]. Further theoretical study is required for the explanation of this behavior, which is out of scope of this experimental Letter.

In existing theories, electron transport in mesoscopic samples is considered within the framework of ballistic, hydrodynamic, or more general models, all based on a detailed approach assuming the solution of the Boltzmann kinetic equation complemented with the boundary conditions for the electron distribution function $[7,12,15-17,23,24,35,41]$. The hydrodynamic description of transport in graphene and other Dirac materials has been mostly focused on the vicinity of the Dirac point, where several anomalies and collective excitations in the Dirac fluid have been predicted [36-39]. Far away from the Dirac point, the system is expected to be similar to an ordinary Fermi liquid $[39,40]$.

Below we make use of the model proposed in Refs. $[6,15,17]$, because it captures all major magnetohydrodynamic properties, including the subtle effects related to the relaxation of the second harmonic of the distribution function by defects and e-e scattering.

The model describes the conductivity as a sum of two independent contributions: The first one is determined by ballistic effects or static disorder and the second one is due to viscosity [15]. This approach assumes the use of the magnetic fielddependent viscosity tensor and the derivation of the resistivity tensor [15],

$$
\rho(B)=\rho_{0}\left(1+\frac{\tau}{\tau^{*}} \frac{1}{1+\left(2 \omega_{c} \tau_{2}\right)^{2}}\right),
$$



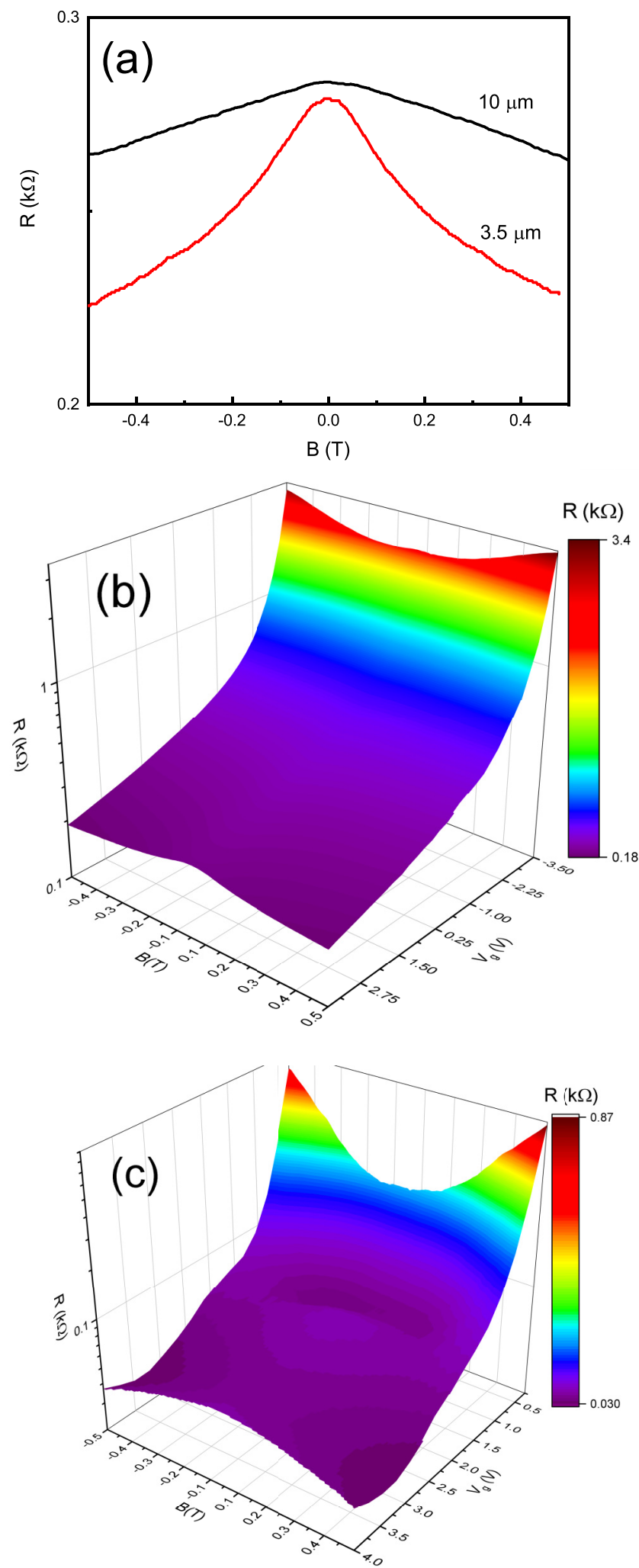

FIG. 2. The resistance $R(B)$ as a function of magnetic field at $V_{g}=4 \mathrm{~V}$ (a) for sample sections of different sizes, and resistance as a function of gate voltage and magnetic field for the sample section widths (b) $W=3.5 \mu \mathrm{m}$ and (c) $W=10 \mu \mathrm{m}, T=4.2 \mathrm{~K}$.

where $\rho_{0}=\frac{m}{e^{2} n \tau}, 1 / \tau$ is the scattering rate due to static disorder, $m$ and $n$ are the effective mass and the density, and

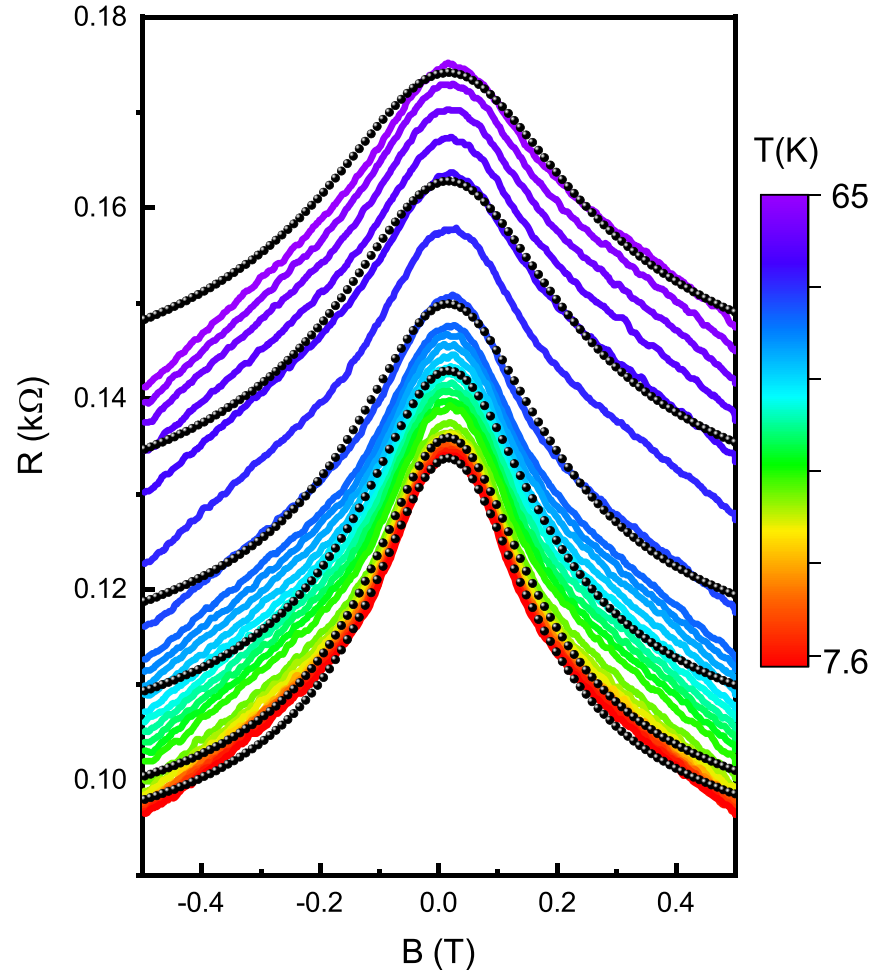

FIG. 3. The resistance $R(B)$ as a function of magnetic field for different temperatures in a sample segment with a width of 3.5 $\mu \mathrm{m}$ for $V_{g}=13 \mathrm{~V}$. The circles show the resistance $R(B)$ calculated from Eq. (1) for different temperatures $T(\mathrm{~K})$ : 7.6, 19.2, 38.5, 46.1, $56.6,64$.

$\tau^{*}=\frac{W\left(W+6 l_{s}\right)}{12 \eta}$, where $\eta=\frac{1}{4} v_{F}^{2} \tau_{2}$ is the viscosity. The shear viscosity relaxation rate is given by $\frac{1}{\tau_{2}(T)}=\frac{1}{\tau_{2, e e}}+\frac{1}{\tau_{2, \text { imp }}}$. The relaxation rate $\frac{1}{\tau_{2, \text { imp }}(T)}$, determined by the process responsible for the relaxation of the second harmonic of the distribution function, such as scattering by static defects, gives rise to viscosity, while $\frac{1}{\tau_{2, e e}(T)}$ refers to the shear viscosity relaxation due to e-e scattering $[6,15]$. The momentum relaxation rate is expressed as $\frac{1}{\tau}=\frac{1}{\tau_{0, \mathrm{ph}}}+\frac{1}{\tau_{0, \mathrm{imp}}}$, where $\tau_{0, \mathrm{ph}}$ is the term responsible for phonon scattering, and $\tau_{0, \text { imp }}$ is the scattering time due to static disorder (not related to the second moment relaxation time) [15]. The boundary conditions can be characterized by a diffusive scattering or by a slip length $l_{s}$ with extreme cases being no-slip $\left(l_{s} \rightarrow 0\right)$ and no-stress $\left(l_{s} \rightarrow \infty\right)$ conditions. It is expected that for $l_{s} \rightarrow \infty$ no hydrodynamic Poiseuille-like flow should be observed.

We fit the magnetoresistance curves and the $R(T)$ at zero magnetic field in Fig. 3 with the three fitting parameters : $\tau(T), \tau^{*}(T)$, and $\tau_{2}(T)$. Note that the $2 \mathrm{D}$ resistivity is proportional to the resistance, $\rho=\frac{W}{L} R$, and below we discuss the resistivity behavior. Figure 3 shows the examples of the calculated Lorentzian-like magnetoresistance at different temperatures. Our experimental data are in good agreement with the theoretical models [6,15]. We have also compared magnetoresistance traces for different gate voltages and extracted the relaxation times versus $N_{s}$ dependences. The corresponding relaxation lengths $l=v_{F} \tau$ and $l_{2}=v_{F} \tau_{2}$ are shown in Fig. 4(a). Note that for massless Dirac fermions the 

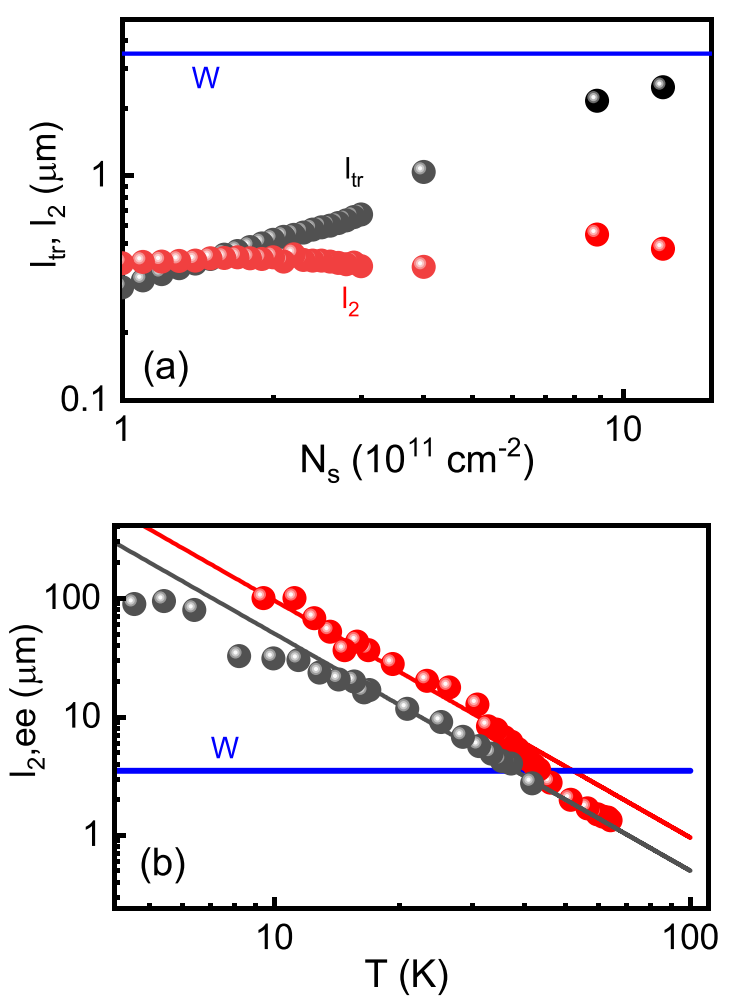

FIG. 4. (a) The relaxations lengths $l_{\mathrm{tr}}$ and $l_{2}$ as a function of the densities, $T=4.2 \mathrm{~K}$. (b) The relaxations length $l_{2, e e}$ as a function of the temperature for two values of the Fermi energy $E_{F}=91 \mathrm{meV}$ (black points) and $E_{F}=114 \mathrm{meV}$ (red points). The thick red and black lines represent theory with parameters indicated in the text. The blue line is the channel width.

Fermi velocity does not depend on the carrier density. One can see that hydrodynamic conditions are approximately met at high densities, where we obtain $l / W \sim 1$ and $l_{2} / W \ll 1$. It is worth noting that the model [6] is valid for $l / W \sim 1$, because two channels providing the relaxation of the second harmonic of the electron distribution function and momentum can be regarded as parallel channels. The transport features associated with the hydrodynamic properties should be observed for $l_{2} / W \ll 1$ and $l_{2} / l \ll 1$, which are reasonably satisfied in our devices at $N_{s}>4.5 \times 10^{11} \mathrm{~cm}^{-2}$ [Fig. 4(a)]. Note, however, that at densities $N_{s}<4.5 \times 10^{12} \mathrm{~cm}^{-2}$, the hydrodynamic conditions are no longer well satisfied in which case both $l_{2}$ and its density dependence become not very meaningful. More stringent conditions $l / W \gg 1$ and $l_{2, e e} / W \ll 1$ lead to a pure hydrodynamic regime, where the second term in Eq. (1) becomes dominant and $\rho \approx \frac{m}{e^{2} n} \eta \frac{12}{W^{2}}$ [7]. In this regime the resistivity is inversely proportional to the square of the temperature, $\rho \sim T^{-2}$, the so-called Gurzhi effect, which has been observed in a high-quality GaAs 2D electron system $[8,27,42]$ and in a graphene quantum point contact $[22,40]$. Because of a lower mobility, in our HgTe zero-gap quantum well the first term in Eq. (1) will dominate, and the Gurzhi effect is suppressed. Another key parameter that determines the hydrodynamic condition is the slip length $l_{s}$, which should be much less than the channel width, $l_{s} / W \ll 1$. From a comparison with the experiment we derive $\tau^{*}$, and the product of $\tau^{*} \tau_{2}=W\left(W+6 l_{s}\right) / 3 v_{F}^{2}$ yields the slip length $l_{s} . l_{s}$ turns out to be negligibly small, confirming the validity of the hydrodynamic description. In addition, this makes it possible to exclude one fitting parameter $\tau^{*}(T)$ from a comparison with the theory, which improves the model readability.

Let us now consider the data on the electron-electron interaction that can be obtained from the processing of magnetoresistance. The interparticle scattering time can be found from the equation

$$
\frac{1}{\tau_{2, e e}(T)}=\frac{1}{\tau_{2}(T)}-\frac{1}{\tau_{2, \mathrm{imp}}}=C \frac{(k T)^{2}}{\hbar E_{F}},
$$

where the numerical factor $C$ would be different for systems with a parabolic and linear spectrum. For a weakly interacting 2D Fermi gas with a parabolic spectrum $C_{\text {par }}$ is defined as [6,26] $C_{\text {par }}=8.4 r_{s}^{2} \ln \left(\frac{1}{k T / E_{F}+r_{s}}\right)$, where the interparticle interaction parameter $r_{s}=1 /\left(a_{B} \sqrt{\pi n}\right)$ is small in a Fermi system, and $a_{B}$ is the Bohr radius. For a system of massless Dirac fermions the parameter $C_{\text {lin }}$ takes the form [5] $C_{\operatorname{lin}} \approx 16.4 \alpha_{e e}^{2}\left[\ln \left(E_{F} / k T\right)\right]$, where the fine-structure constant $\alpha_{e e}$ is defined as $\alpha_{e e}=\frac{e^{2}}{\varepsilon \hbar v_{F}}$ and $\varepsilon$ is the dielectric constant. In Dirac systems the dimensionless parameter and the finestructure constant have the same value. In the $\mathrm{HgTe} \mathrm{QW}$ we find $\alpha_{e e}=r_{s}=3.2 / \varepsilon$.

Comparing the temperature dependence of the relaxation rate $1 / \tau_{2, e e}(T)$ with Eq. (2), we can derive a temperatureindependent characteristic time $\tau_{2, \text { imp }}=0.65 \times 10^{-12} \mathrm{~s}$. The hydrodynamic approach is associated with a substantial relaxation of the $m$ th harmonic of the distribution function due to disorder scattering with the rates $\tau_{m \text {,imp }}$ [6]. It is usually argued that $\tau=\tau_{1, \text { imp }}[6,41]$ and $\tau_{2, \text { imp }}$ are of the same order of magnitude. In our sample we obtain $\tau_{1, \text { imp }}=4.2 \times 10^{-12} \mathrm{~s}>$ $\tau_{2, \text { imp }}$. Note that in high-mobility GaAs quantum wells it has been found that the time $\tau=\tau_{1, \text { imp }}$ is much longer than $\tau_{2, \text { imp }}$ (by 10-100 times) $[6,27,42]$.

Figure 4(b) shows the mean free path $l_{2, e e}=v_{F} \tau_{2, e e}$ vs $T$ in a broad temperature range for two values of the Fermi energy $E_{F}=91 \mathrm{meV}$ and $E_{F}=114 \mathrm{meV}$. Comparing our results with Eq. (2) we find parameters $C=1.1$ and $C=0.7$ for lower and higher energies. Thus, a comparison with theory turned out to be possible when the temperature changes by one order of magnitude. These values are very different from the values obtained for a $2 \mathrm{D}$ electron Fermi system in a GaAs well, which are in the order of 5-7 [6,27,41,42]. Parameters $C$ obtained from the experiment coincide in order of magnitude with the results of the calculation, if we assume the dimensionless parameter $r_{s} \sim 0.1-0.15$ which is very different from graphene $\left(r_{s} \approx 0.7\right)$ due to a high dielectric constant. Although the equations for $C_{\text {par }}$ and $C_{\text {lin }}$ look different due to the logarithmic term, the differences would not be enough to be significant in the temperature dependence. The discrimination of the subtle difference between a massive and massless spectrum is still a challenging task and requires more experimental and theoretical work.

In summary, we have performed a detailed study of the magnetotransport in a single-cone massless Dirac fermion system. The negative magnetoresistance was fitted by a 
Lorentzian profile in accordance with the hydrodynamic approach and the shear stress relaxation time was extracted, which determines the viscosity in the Fermi liquid with a linear dispersion. Compared to graphene, the most typical Dirac material, our system has a number of advantages. First, the fact that only one valley is present in the spectrum allows for a more unambiguous interpretation of the transport measurements, whereas the intervalley scattering present in graphene can affect boundary scattering. Second, the advantage of a simpler manufacturing method (MBE growth versus exfoliation) allows for the fabrication of samples of macroscopic size and, therefore, for discriminating between the transport properties due to a Poiseuille flow in narrow channels and those due to scattering in the bulk.

The financial support of this work by the Ministry of Science and Higher Education of the Russian Federation, Grant No. 075-15-2020- 797 (13.1902.21.0024). São Paulo Research Foundation (FAPESP) Grant No. 2015/16191-5, and the National Council for Scientific and Technological Development $(\mathrm{CNPq})$ is acknowledged. We thank O. E. Raichev for helpful discussions.
[1] J. J. Quinn and R. A. Ferrell, Electron self-energy approach to correlation in a degenerate electron gas, Phys. Rev. 112, 812 (1958).

[2] A. Chaplik, Energy spectrum and electron scattering processes in inversion layers, Zh. Eksp. Teor. Fiz. 60, 1845 (1971) [Sov. Phys. JETP 33, 997 (1971)].

[3] G. F. Giuliani and J. J. Quinn, Lifetime of a quasiparticle in a two-dimensional electron gas, Phys. Rev. B 26, 4421 (1982).

[4] A. Principi, G. Vignale, M. Carrega, and M. Polini, Bulk and shear viscosities of the two-dimensional electron liquid in a doped graphene sheet, Phys. Rev. B 93, 125410 (2016).

[5] B. N. Narozhny and M. Schutt, Magnetohydrodynamics in graphene: Shear and Hall viscosities, Phys. Rev. B 100, 035125 (2019).

[6] P. S. Alekseev and A. P. Dmitriev, Viscosity of two-dimensional electrons, Phys. Rev. B 102, 241409(R) (2020).

[7] R. N. Gurzhi, Minimum of resistance in impurity-free conductors, Sov. Phys. JETP 44, 771 (1963) [Sov. Phys. Usp. 11, 255 (1968)].

[8] M. J. M. de Jong and L. W. Molenkamp, Hydrodynamic electron flow in high-mobility wires, Phys. Rev. B 51, 13389 (1995).

[9] M. Müller, J. Schmalian, and L. Fritz, Graphene: A Nearly Perfect Fluid, Phys. Rev. Lett. 103, 025301 (2009).

[10] A. V. Andreev, S. A. Kivelson, and B. Spivak, Hydrodynamic Description of Transport in Strongly Correlated Electron Systems, Phys. Rev. Lett. 106, 256804 (2011).

[11] B. N. Narozhny, I. V. Gornyi, M. Titov, M. Schütt, and A. D. Mirlin, Hydrodynamics in graphene: Linear-response transport, Phys. Rev. B 91, 035414 (2015).

[12] I. Torre, A. Tomadin, A. K. Geim, and M. Polini, Nonlocal transport and the hydrodynamic shear viscosity in graphene, Phys. Rev. B 92, 165433 (2015).

[13] D. A. Bandurin, I. Torre, R. K. Kumar, M. Ben Shalom, A. Tomadin, A. Principi, G. H. Auton, E. Khestanova, K. S. Novoselov, I. V. Grigorieva, L. A. Ponomarenko, A. K. Geim, and M. Polini, Negative local resistance caused by viscous electron backflow in graphene, Science 351, 1055 (2016).

[14] R. K. Kumar, D. A. Bandurin, F. M. D. Pellegrino, Y. Cao, A. Principi, H. Guo, G. H. Auton, M. B. Shalom, L. A. Ponomarenko, G. Falkovich, K. Watanabe, T. Taniguchi, I. V. Grigorieva, L. S. Levitov, M. Polini, and A. K. Geim, Superballistic flow of viscous electron fluid through graphene constrictions, Nat. Phys. 13, 1182 (2017).

[15] P. S. Alekseev, Negative Magnetoresistance in Viscous Flow of Two-Dimensional Electrons, Phys. Rev. Lett. 117, 166601 (2016).
[16] A. Lucas, J. Crossno, K. C. Fong, P. Kim, and S. Sachdev, Transport in inhomogeneous quantum critical fluids and in the Dirac fluid in graphene, Phys. Rev. B 93, 075426 (2016).

[17] T. Scaffidi, N. Nandi, B. Schmidt, A. P. Mackenzie, and J. E. Moore, Hydrodynamic Electron Flow and Hall Viscosity, Phys. Rev. Lett. 118, 226601 (2017).

[18] A. Levchenko, H.-Y. Xie, and A. V. Andreev, Viscous magnetoresistance of correlated electron liquids, Phys. Rev. B 95 , 121301(R) (2017).

[19] A. Lucas, Stokes paradox in electronic Fermi liquids, Phys. Rev. B 95, 115425 (2017).

[20] D. A. Bandurin, A. V. Shytov, L. S. Levitov, R. Krishna Kumar, A. I. Berdyugin, M. Ben Shalom, I. V. Grigorieva, A. K. Geim, and G. Falkovich, Fluidity onset in graphene, Nat. Commun. 9, 4533 (2018).

[21] A. I. Berdyugin, S. G. Xu, F. M. D. Pellegrino, R. Krishna Kumar, A. Principi, I. Torre, M. Ben Shalom, T. Taniguchi, K. Watanabe, I. V. Grigorieva, M. Polini, A. K. Geim, and D. A. Bandurin, Measuring Hall viscosity of graphene's electron fluid, Science 364, 162 (2019).

[22] M. Kim, S. G. Xu, A. I. Berdyugin, A. Principi, S. Slizovskiy, N. Xin, P. Kumaravadivel, W. Kuang, M. Hamer, R. Krishna Kumar, R. V. Gorbachev, K. Watanabe, T. Taniguchi, I. V. Grigorieva, V. I. Falko, M. Polini, and A. K. Geim, Control of electron-electron interaction in graphene by proximity screening, Nat. Commun. 11, 2339 (2020).

[23] A. Lucas and S. A. Hartnoll, Kinetic theory of transport for inhomogeneous electron fluids, Phys. Rev. B 97, 045105 (2018).

[24] F. M. D. Pellegrino, I. Torre, and M. Polini, Nonlocal transport and the Hall viscosity of two-dimensional hydrodynamic electron liquids, Phys. Rev. B 96, 195401 (2017).

[25] E. I. Kiselev and J. Schmalian, Boundary conditions of viscous electron flow, Phys. Rev. B 99, 035430 (2019).

[26] D. S. Novikov, Viscosity of a two-dimensional Fermi liquid, arXiv:cond-mat/0603184.

[27] G. M. Gusev, A. D. Levin, E. V. Levinson, and A. K. Bakarov, Viscous electron flow in mesoscopic two-dimensional electron gas, AIP Adv. 8, 025318 (2018).

[28] G. M. Gusev, A. D. Levin, E. V. Levinson, and A. K. Bakarov, Viscous transport and Hall viscosity in a two-dimensional electron system, Phys. Rev. B 98, 161303(R) (2018).

[29] A. D. Levin, G. M. Gusev, E. V. Levinson, Z. D. Kvon, and A. K. Bakarov, Vorticity-induced negative nonlocal resistance in a viscous two-dimensional electron system, Phys. Rev. B 97 245308 (2018). 
[30] G. M. Gusev, A. S. Jaroshevich, A. D. Levin, Z. D. Kvon, and A. K. Bakarov, Stokes flow around an obstacle in viscous twodimensional electron liquid, Sci. Rep. 10, 7860 (2020).

[31] P. S. Alekseev and M. A. Semina, Ballistic flow of twodimensional interacting electrons, Phys. Rev. B 98, 165412 (2018).

[32] P. S. Alekseev and M. A. Semina, Hall effect in a ballistic flow of two-dimensional interacting particles, Phys. Rev. B 100, 125419 (2019).

[33] M. Chandra, G. Kataria, D. Sahdev, and R. Sundararaman, Hydrodynamic and ballistic AC transport in two-dimensional Fermi liquids, Phys. Rev. B 99, 165409 (2019).

[34] I. S. Burmistrov, M. Goldstein, M. Kot, V. D. Kurilovich, and P. D. Kurilovich, Dissipative and Hall Viscosity of a Disordered 2D Electron Gas, Phys. Rev. Lett. 123, 026804 (2019).

[35] T. Holder, R. Queiroz, T. Scaffidi, N. Silberstein, A. Rozen, J. A. Sulpizio, L. Ella, S. Ilani, and A. Stern, Ballistic and hydrodynamic magnetotransport in narrow channels, Phys. Rev. B 100, 245305 (2019).

[36] U. Briskot, M. Schutt, I. V. Gornyi, M. Titov, B. N. Narozhny, and A. D. Mirlin, Collision-dominated nonlinear hydrodynamics in graphene, Phys. Rev. B 92, 115426 (2015).

[37] D. Svintsov, Hydrodynamic-to-ballistic crossover in Dirac materials, Phys. Rev. B 97, 121405(R) (2018).

[38] O. Kashuba, B. Trauzettel, and L. W. Molenkamp, Relativistic Gurzhi effect in channels of Dirac materials, Phys. Rev. B 97, 205129 (2018).

[39] A. Lucas and K. C. Fong, Hydrodynamics of electrons in graphene, J. Phys.: Condens. Matter 30, 053001 (2018).
[40] M. Polini and A. K. Geim, Viscous electron fluids, Phys. Today 73(6), 28 (2020).

[41] O. E. Raichev, G. M. Gusev, A. D. Levin, and A. K. Bakarov, Manifestations of classical size effect and electronic viscosity in the magnetoresistance of narrow two-dimensional conductors: Theory and experiment, Phys. Rev. B 101, 235314 (2020).

[42] G. M. Gusev, A. S. Jaroshevich, A. D. Levin, Z. D. Kvon, and A. K. Bakarov, Viscous magnetotransport and Gurzhi effect in bilayer electron system, Phys. Rev. B 103, 075303 (2021).

[43] B. Büttner, C. X. Liu, G. Tkachov, E. G. Novik, C. Brüne, H. Buhmann, E. M. Hankiewicz, P. Recher, B. Trauzettel, S. C. Zhang, and L. W. Molenkamp, Single valley Dirac fermions in zero-gap HgTe quantum wells, Nat. Phys. 7, 418 (2011).

[44] D. A. Kozlov, Z. D. Kvon, N. N. Mikhailov, and S. A. Dvoretskii, Weak localization of Dirac fermions in HgTe quantum wells, JETP Lett. 96, 730 (2012).

[45] See Supplemental Material at http://link.aps.org/supplemental/ 10.1103/PhysRevResearch.3.L032031 for details on the magnetoresistance measurements in macroscopic and mesoscopic HgTe wells.

[46] A. A. Dobretsova, Z. D. Kvon, L. S. Braginskii, M. V. Entin, and N. N. Mikhailov, Mobility of Dirac electrons in HgTe quantum wells, JETP Lett. 104, 388 (2016).

[47] Ye. O. Melezhik, J. V. Gumenjuk-Sichevska, and F. F. Sizov, Electron relaxation and mobility in the inverted band quantum well $\mathrm{CdTe} / \mathrm{Hg}_{1-x} \mathrm{Cd}_{x} \mathrm{Te} / \mathrm{CdTe}$, Semicond. Phys., Quantum Electron. Optoelectron. 17, 85 (2014). 